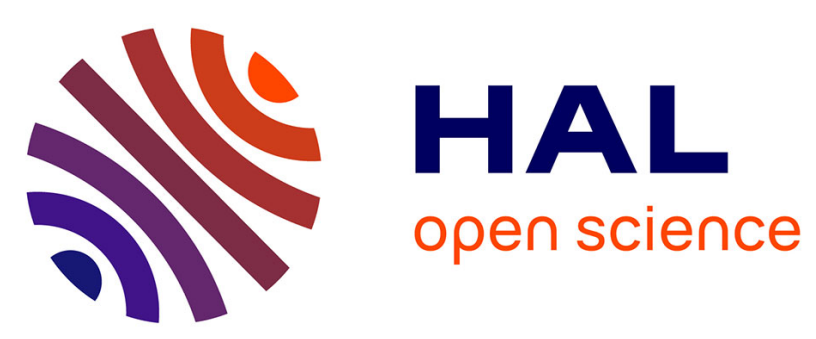

\title{
Guard Time Optimisation for Energy Efficiency in IEEE 802.15.4-2015 TSCH Links
}

Georgios Papadopoulos, Alexandros Mavromatis, Xenofon Fafoutis, Robert

Piechocki, Theo Tryfonas, George Oikonomou

\section{- To cite this version:}

Georgios Papadopoulos, Alexandros Mavromatis, Xenofon Fafoutis, Robert Piechocki, Theo Tryfonas, et al.. Guard Time Optimisation for Energy Efficiency in IEEE 802.15.4-2015 TSCH Links. InterIoT 2016 : 2nd EAI International Conference on Interoperability in IoT , Oct 2016, Paris, France. pp.56 $63,10.1007 / 978-3-319-52727-7 \_8$. hal-01574829

\section{HAL Id: hal-01574829 \\ https://hal.science/hal-01574829}

Submitted on 16 Aug 2017

HAL is a multi-disciplinary open access archive for the deposit and dissemination of scientific research documents, whether they are published or not. The documents may come from teaching and research institutions in France or abroad, or from public or private research centers.
L'archive ouverte pluridisciplinaire HAL, est destinée au dépôt et à la diffusion de documents scientifiques de niveau recherche, publiés ou non, émanant des établissements d'enseignement et de recherche français ou étrangers, des laboratoires publics ou privés. 


\title{
Guard Time Optimisation for Energy Efficiency in IEEE 802.15.4-2015 TSCH Links
}

\author{
Georgios Z. Papadopoulos ${ }^{1,2}$, Alexandros Mavromatis ${ }^{1}$, Xenofon Fafoutis ${ }^{1}$, \\ Robert Piechocki ${ }^{1}$, Theo Tryfonas ${ }^{1}$, and George Oikonomou ${ }^{1}$ \\ 1 Faculty of Engineering, University of Bristol, UK, \\ [a.mavromatis, xenofon.fafoutis, r.j.piechocki, \\ theo.tryfonas, g. oikonomou] @bristol.ac.uk \\ 2 IRISA, Télécom Bretagne, Institut Mines-Télécom, France, \\ georgios.papadopoulos@telecom-bretagne.eu
}

\begin{abstract}
Time Slotted Channel Hopping (TSCH) is among the Medium Access Control (MAC) schemes defined in the IEEE 802.15.4-2015 standard. TSCH aims to guarantee high-level network reliability by keeping nodes time-synchronised. In order to ensure successful communication between a sender and a receiver, the latter starts listening shortly before the expected time of a MAC layer frame's arrival. The offset between the time a node starts listening and the estimated time of frame arrival is called guard time and it aims to reduce the probability of missed frames due to clock drift. In this paper, we investigate the impact of the guard time length on network performance. We identify that, when using the $6 \mathrm{TiSCH}$ minimal schedule, the most significant cause of energy consumption is idle listening during guard time. Therefore, we perform empirical optimisations on the guard time to maximise the energy-efficiency of a TSCH link. Our experiments, conducted using the Contiki OS, show that optimal guard time configuration can reduce energy consumption by up to $40 \%$, without compromising network reliability.
\end{abstract}

Key words: Internet of Things, IEEE 802.15.4-2015, TSCH, Synchronisation, Guard Time, Performance Evaluation, Energy Consumption.

\section{Introduction}

In 2016 the IEEE 802.15.4-2015 standard [1] was published to offer a certain quality of service for deterministic industrial-type applications. Among the operating modes defined in this standard, Time-Slotted Channel Hopping (TSCH) is a Medium Access Control (MAC) protocol for low-power and reliable networking solutions in Low-Power Lossy Networks (LLNs). Although there is a vast literature of unstandardised MAC protocols that are optimised for different scenarios [2], the standardised TSCH offers interoperability between IoT devices. TSCH specifies a channel hopping scheme to avoid interference, and consequently to enable high reliability [3], while it employs time synchronisation to achieve low-power operation (Fig. 1). TSCH presents a deterministic 


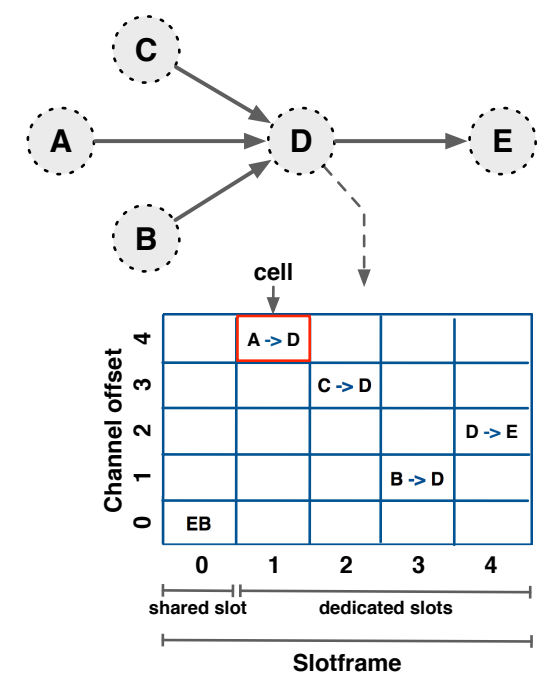

Fig. 1: An example TSCH schedule for node D. $A>D$ stands for "node A sends to node D", while $E B$ cells are used for broadcast and advertisement frames.

scheduling approach where each cell consists of a pair of a timeslot and a channel offset for collision avoidance purposes. Each channel offset is translated into a frequency through a function that uses as input the ASN (Absolute Sequence Number) and the number of available frequencies (e.g., 16 when using IEEE 802.15.4-compliant radios at $2.4 \mathrm{GHz}$ with all channels in use) [4].

To account for loss of synchronisation, a TSCH receiver maintains its radio on receiving mode for an extended period of time, named Guard Time. In [5], we highlighted the effect of guard time on network performance. We identified that, when employing the $6 \mathrm{TiSCH}$ minimal schedule, most of the energy consumed is wasted in idle listening, due to the guard time. In this paper, we further investigate the importance of guard time optimisation. To this aim, we study using both an analytical model and simulations the optimal guard time as a function of the clock drift. Our performance evaluation results using the Cooja simulator, demonstrate that fine-tuning the guard time, under realistic clock drift configurations (e.g., 20 ppm, $30 \mathrm{ppm}$ ), can significantly improve the energy efficiency of a TSCH link without compromising its reliability.

\section{TSCH Overview}

Under the TSCH scheme, nodes periodically exchange Enhanced Beacon (EB) packets to remain time-synchronised throughout the network's lifetime. Synchronisation does not need explicit EB exchange, data packets may also be utilised to compute clock drifts [6]. Typically, an EB contains time and channel frequency information, as well as information about the initial link and slotframe for new nodes to join the network. New nodes may join a TSCH network by "hearing" an EB frame from another node. 


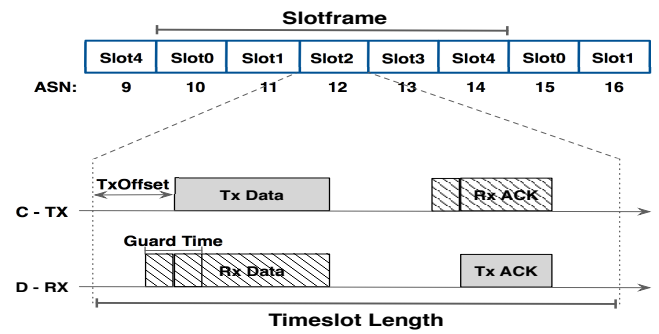

Fig. 2: A typical TSCH timeslot template for a transmitter (top) and receiver node (bottom): node $\mathrm{C}$, transmits its data packet after TxOffset, while the receiver $\mathrm{D}$, uses a Guard Time to avoid missing the incoming packet by turning its radio on slightly before the packet arrival.

Fig. 2 illustrates a typical TSCH-based communication between two nodes. In TSCH networks, time is divided into timeslots of equal length, large enough to transmit a frame and to receive an acknowledgement, while a set of timeslots construct a slotframe. At each timeslot, a node may transmit or receive a frame, or it may turn its radio off for saving energy. Each timeslot can be either dedicated (contention-free) or shared (contention-based approach). Finally, each timeslot is labelled with ASN, a variable which counts the number of timeslots since the network was established, ASN is initialised to 0 .

A node transmits a data packet at the beginning of each timeslot, exactly after the TxOffset. TSCH incorporates a Guard Time to account for loss of synchronisation. To account for both positive and negative clock drift, the receiver wakes up before the expected end of the TxOffset and keeps the radio on for $\tau$ seconds or until a frame preamble is received. The guard time $\tau$ is equally spaced around the end of the TxOffset. Thus, for a certain guard time, $\tau$, the maximum synchronisation error, $\epsilon_{\tau}$, that can be tolerated is:

$$
\epsilon_{\tau}=\frac{\tau}{2}-\tau_{p},
$$

where $\tau_{p}$ is the time required for the reception of the frame preamble. Let us consider the use of clocks with an error of $\pm e_{f}$. The synchronisation error accumulates over time. The worst case scenario for synchronisation is right before a synchronisation event (e.g., EB frame), when the error is:

$$
\epsilon_{T}=T\left(\frac{1}{1-e_{f}}-\frac{1}{1+e_{f}}\right),
$$

where $T$ is the period of synchronisation events. By equating (1) and (2), we calculate a minimum guard time required to achieve zero packet loss due to loss of synchronisation $\left(\tau_{m}\right)$ :

$$
\tau_{m}=2 T\left(\frac{1}{1-e_{f}}-\frac{1}{1+e_{f}}\right)+2 \tau_{p} .
$$

It can be observed that in the ideal case where the clock error is $e_{f}=0 \mathrm{ppm}$, the minimum acceptable guard time is $\tau_{m}=2 \tau_{p}$. 


\begin{tabular}{ll}
\hline \hline Topology parameters & Value \\
\hline Number of nodes & 2 (a transmitter and receiver $)$ \\
Node spacing & $20 \mathrm{~m}$ in a line topology \\
\hline Simulation parameters Value \\
\hline Duration & 60 minutes \\
Traffic Pattern & 1 frame / $60 \mathrm{~s}$ \\
Data packet size & 102 bytes $(77$ bytes payload $)$ \\
Routing model & $\mathrm{RPL}[7]$ \\
MAC model & TSCH $(6 \mathrm{TiSCH}$ minimal schedule $)$ \\
\hline TSCH parameters & Value \\
\hline EB period & $3.42 \mathrm{~s}$ \\
Slotframe length & 7 \\
Timeslot length & $15 \mathrm{~ms}$ \\
Guard Time & $(0-2200)$ us \\
Clock Drift & $(0, \pm 10, \pm 20, \pm 30$ and \pm 40$) \mathrm{ppm}$ \\
\hline Hardware parameters & Value \\
\hline Antenna model & $\mathrm{CC} 2420$ \\
Radio propagation & $2.4 \mathrm{GHz}$ \\
Transmission power & $0 \mathrm{dBm}$ \\
\hline \hline
\end{tabular}

Table 1: Simulation setup.

\section{Performance evaluation}

In order to assess the impact of guard time in the performance of TSCH, we performed a set of experiments using Cooja, the network simulator distributed as part of the Contiki open-source operating system for the Internet of Things ${ }^{1}$. In our experiments we emulated Z1 motes. We conducted a large number of simulations under various realistic clock drifts (e.g., $\pm 10, \pm 20$ ppm). To account for the worst case scenario, we configured the transmitter node to the maximum positive clock drift and the receiver at the maximum negative drift. For instance, in the case of the $\pm 20 \mathrm{ppm}$ configuration, we set the transmitter node at $+20 \mathrm{ppm}$ and the receiver at $-20 \mathrm{ppm}$, resulting to a relative drift of $40 \mathrm{ppm}$. The clock drifts are constant throughout each simulation. Furthermore, we performed simulations under different guard time (e.g., 400, 600 us) configurations, while keeping the default values for the remaining parameters, such as $\mathrm{EB}$ or data packet transmission frequency.

\subsection{Setup}

For our evaluation we use a scenario with two nodes, one leaf transmitter and one sink receiver, positioned at a distance of $20 \mathrm{~m}$. We choose the data packet size to be equal to 102 bytes that corresponds to all necessary information for MAC, routing and application operations. Furthermore, we use Cooja's Unit Disk Graph Medium (UDGM) radio model, with each node transmitting frames at $0 \mathrm{dBm}$. Lastly, each simulation lasted $60 \mathrm{~min}$. Full details of the simulation setup are presented in Table 1 .

\footnotetext{
${ }^{1}$ Contiki OS - www.contiki-os.org
} 

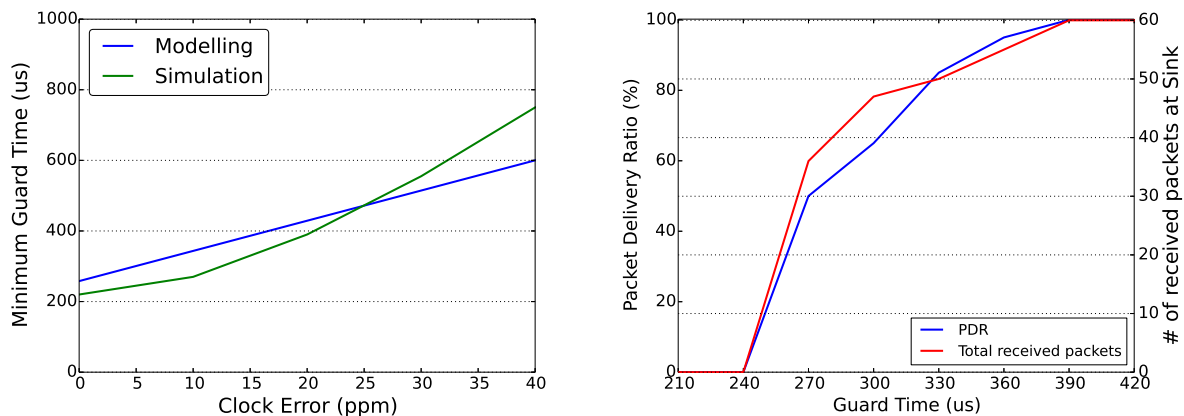

(a) Minimum guard time for operation (b) PDR \& goodput performance under a without packet loss. \pm 20 ppm clock drift.

Fig. 3: Minimum required guard time for various clock drifts (left) and the network performance under $\pm 20 \mathrm{ppm}$ clock drift (right), values are in average.

\subsection{Simulation Results}

In [5], we studied the impact of idle listening during guard time on energy consumption. Hereinafter we discuss our proposed guard time optimisation and the gains that it can offer in terms of reliability, goodput and energy consumption.

Guard Time: We first investigate the minimum guard time, while guaranteeing 100\% Packet Delivery Ratio (PDR), under different clock drift values (i.e., $0, \pm 10, \pm 20, \pm 30$ and $\pm 40 \mathrm{ppm}$ ) using both the analytical model and a set of simulations. Note that packet loss is calculated as $1-P D R$, and thus, packet loss $0 \%$ is the equivalent of $100 \%$ PDR. As can be observed from Fig. 3a, Eq. (3) approximates a linear behaviour $\left(\tau_{p}=129 u s, T=1.71 \mathrm{~s}\right)$, which is validated by the simulations. For instance, in case of a $\pm 20 \mathrm{ppm}$ drift, a typical worstcase clock drift in IoT-devices [8], 390 us is the minimum guard time length for operation without compromising network reliability due to loss of synchronisation or goodput (Fig. 3b). Note that both nodes operate as EB transmitters and receivers; thus, the link is synchronised at half the EB period on average, $T=3.42 / 2=1.71 \mathrm{~s}$.

Energy Efficiency To evaluate the energy consumption of each network node, we employed Contiki's Powertrace and Energest modules. These modules monitor and log the radio and Micro-Controller Unit (MCU) usage in real-time by tracking the time spent in various states (i.e., Radio transmitting or receiving, or sleeping). Table 2 provides typical current consumption levels at each of these states for the $\mathrm{Z} 1$ mote $^{2}$, under a $3 \mathrm{~V}$ operating voltage. Note that in this evaluation we focus on the energy consumption performance related with the radio communication only.

\footnotetext{
${ }^{2}$ http://zolertia.sourceforge.net/wiki/images/e/e8/Z1_RevC_Datasheet.pdf
} 


\begin{tabular}{llr}
\hline \hline IC & Notes & Current Consumption \\
\hline CC2420 TX Mode @ $0 \mathrm{dBm}$ & $17.4 \mathrm{~mA}$ \\
& RX Mode & $18.8 \mathrm{~mA}$ \\
& $0.5 \mathrm{uA}$ \\
\hline
\end{tabular}

Table 2: Approximate energy consumption of the $\mathrm{Z} 1$ mote.

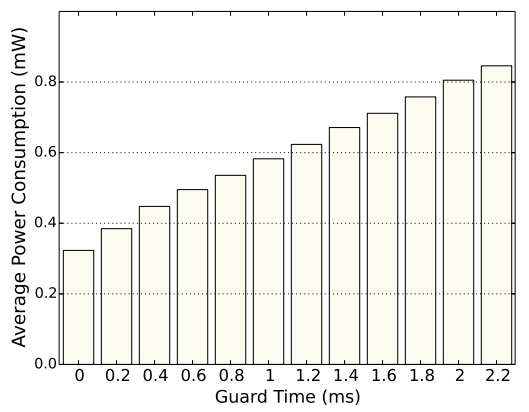

(a) Average power consumption, under different guard time durations.

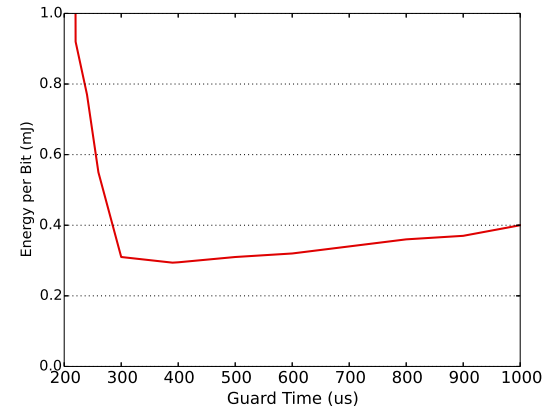

(b) Energy consumption per successful received bit.

Fig. 4: A thorough power consumption performance of the TSCH scheme, under $\mathrm{a} \pm 20$ ppm clock drift [8].

We here investigate the impact of guard time duration on energy consumption $( \pm 20 \mathrm{ppm})$. To this aim, we first present energy consumption performance under various guard time configurations. Our results demonstrate that by reducing guard time (i.e., from 2200 us, the default configuration of Contiki's TSCH implementation, to $400 \mathrm{us}$ ), we can decrease the average power consumption per node (i.e., receiver node in our scenario) by more than 40\%, (Fig. 4a). Energy consumption is reduced further at guard times lower than 390 us, yet at the cost of compromising reliability. To better visualise this trade-off, we define the energy-efficiency of TSCH as the average energy consumed for the successful reception of a single bit, and it is calculated as follows:

$$
\eta=\frac{E}{P D R * T_{\text {transmissions }} * P_{\text {size }} * 8},
$$

where $E$ is the total energy consumed during the experiment, $T_{\text {transmissions }}$ is the total count of frame transmissions from the leaf to the sink node, while $P_{\text {size }}$ is the size of a data frame in bytes. Fig. $4 \mathrm{~b}$ plots the energy efficiency of TSCH as a function of guard time. It can be observed that there is an optimisation point for the guard time at 390 us. Below that optimal configuration the energy per correct bit increases rapidly, due to packet loss caused by loss of synchronisation. Above that optimal configuration the energy per correct bit increases again, as the energy consumed in idle listening increases with the guard time. 


\section{Conclusion}

In this work, we first investigated the impact of guard time on TSCH performance in terms of network reliability, goodput and energy consumption. We then performed empirical optimisations on the guard time to maximise the energyefficiency of a TSCH link. Our performance evaluation results, using the Cooja simulator, demonstrate that the guard time has a straightforward impact on energy consumption. In particular, we have shown that fine-tuning the guard time can result into significant savings in energy consumption without compromising network reliability. Our ongoing work consists of further investigating this lead in multi-hop networks, where the clock drift may have a heavy impact on networkwide time synchronisation. Furthermore, we plan to study the behaviour of TSCH under realistic conditions by performing a set of experimental studies over the FIT IoT-LAB testbed [9].

\section{Acknowledgements}

This work was partially performed under IRC-SPHERE funded by EPSRC, Grant EP/K031910/1.

\section{References}

1. "IEEE Standard for Low-Rate Wireless Personal Area Networks (LR-WPANs)," IEEE Std 802.15.4-2015 (Revision of IEEE Std 802.15.4-2011), April 2016.

2. A. Bachir, M. Dohler, T. Watteyne, and K. Leung, "MAC Essentials for Wireless Sensor Networks," Communications Surveys Tutorials, IEEE, vol. 12, no. 2, pp. 222-248, Second 2010.

3. T. Watteyne, A. Mehta, and K. Pister, "Reliability Through Frequency Diversity: Why Channel Hopping Makes Sense," in Proceedings of the 6th ACM Symposium on Performance Evaluation of Wireless Ad Hoc, Sensor, and Ubiquitous Networks, 2009, pp. 116-123.

4. T. Watteyne, M. Palattella, and L. Grieco, "Using IEEE 802.15.4e Time-Slotted Channel Hopping (TSCH) in the Internet of Things (IoT): Problem Statement," RFC 7554, 2015.

5. A. Mavromatis, G. Z. Papadopoulos, X. Fafoutis, A. Elsts, G. Oikonomou, and T. Tryfonas, "Impact of Guard Time Length on IEEE 802.15.4e TSCH Energy Consumption," in Proceedings of the IEEE International Conference on Sensing, Communication and Networking (SECON), 2016.

6. T. Chang, T. Watteyne, K. Pister, and Q. Wang, "Adaptive Synchronization in Multi-hop TSCH Networks," Computer Networks, vol. 76, pp. 165-176, 2015.

7. T. Winter, P. Thubert, A. Brandt, J. Hui, R. Kelsey, P. Levis, K. Pister, R. Struik, J. Vasseur, and A. R., "RPL: IPv6 Routing Protocol for Low-Power and Lossy Networks," RFC 6550, 2012.

8. X. Fafoutis, B. Janko, E. Mellios, G. Hilton, R. S. Sherratt, R. Piechocki, and I. Craddock, "SPW-1: A Low-Maintenance Wearable Activity Tracker for Residential Monitoring and Healthcare Applications," in Proceedings of the EAI International Conference on Wearables in Healthcare (HealthWear), 2016.

9. G. Z. Papadopoulos, J. Beaudaux, A. Gallais, T. Noel, and G. Schreiner, "Adding value to WSN simulation using the IoT-LAB experimental platform," in Proceedings of the 9th IEEE International Conference on Wireless and Mobile Computing, Networking and Communications (WiMob), 2013, pp. 485-490. 